Honam Mathematical J. 32 (2010), No. 1, pp. 167-176

\title{
ON STABILITY OF EINSTEIN WARPED PRODUCT MANIFOLDS
}

\author{
Yong-Soo Pyo, Hyun Woong Kim And Joon-Sik PARK
}

\begin{abstract}
Let $(B, \breve{g})$ and $(N, \hat{g})$ be Einstein manifolds. Then, we get a complete (necessary and sufficient) condition for the warped product manifold $B \times{ }_{f} N:=(B \times N, \check{g}+f \hat{g})$ to be Einstein, and obtain a complete condition for the Einstein warped product manifold $B \times{ }_{f} N$ to be weakly stable. Moreover, we get a complete condition for the map $i:(B, \breve{g}) \times(N, \hat{g}) \rightarrow B \times{ }_{f} N$, which is the identity map as a map, to be harmonic. Under the assumption that $i$ is harmonic, we obtain a complete condition for $B \times{ }_{f} N$ to be Einstein.
\end{abstract}

\section{Introduction}

A harmonic map $\phi$ from a compact Riemannian manifold $(M, g)$ into another Riemannian manifold $(N, h)$ is a critical point of the energy functional $([5,9])$

$$
E(\phi):=\int_{M} e(\phi) v_{g},
$$

where $e(\phi)=\frac{1}{2} h(d \phi, d \phi)$. The second variation formula of the energy functional $E$ for a harmonic map $\phi$ is given as follows ([6, 8, 9]):

$$
H(E)_{\phi}(V, V):=\left.\frac{d^{2}}{d t^{2}} E\left(\phi_{t}\right)\right|_{t=0}=\int_{M} h\left(V, J_{\phi} V\right) v_{g}
$$

Received September. 24, 2009. Accepted March. 12, 2010.

2000 Mathematics Subject Classification: 53C07.

Key words and phrases: harmonic map, stability of harmonic map, warped product manifold.

Corresponding author: Yong-Soo Pyo. 
where $J_{\phi}$ is the Jacobi operator acting on $\Gamma\left(\phi^{-1} T N\right)$ and

$$
V_{p}:=\left.\left(d \phi_{t}(p) / d t\right)\right|_{t=0}, \quad p \in M .
$$

Then $\phi$ is said to be stable (resp. weakly stable) if $H(E)_{\phi}(V, V)>0$ (resp. $\geq 0$ ) for all $V \in \Gamma\left(\phi^{-1} T N\right)$, and otherwise, is said to be unstable.

To construct a harmonic map between two Riemannian manifolds and to show the stability of a given harmonic map are very important topics in the study on the theory of harmonic maps.

In this paper, let $(B, \check{g})$ and $(N, \hat{g})$ be two Riemannian manifolds. Then, $B \times{ }_{f} N:=(B \times N, \check{g}+f \hat{g})$, $\left(f\right.$ being a positive $C^{\infty}$-function on $B)$, is said to be a warped product manifold $([1])$ of $(B, \check{g})$ and $(N, \hat{g})$. We assume that $(B, \check{g})$ and $(N, \hat{g})$ are compact Einstein manifolds. Then, we obtain a necessary and sufficient condition for $B \times{ }_{f} N$ to be Einstein (cf. Theorem 2.3). And then, using R. T. Smith's Stability Theorem, we get a necessary and sufficient condition for such an Einstein warped product manifold to be weakly stable (cf. Theorem 2.5), and get a sufficient condition for the Einstein warped product manifold to be stable (cf. Corollary 2.6). Moreover, we get a complete condition (cf. Proposition 2.1) for the map $i:(B, \check{g}) \times(N, \hat{g}) \rightarrow B \times_{f} N$, being the identity map as a map, to be harmonic. Under the assumption that $i$ is harmonic, we obtain a complete condition (cf. Theorem 2.2) for $B \times{ }_{f} N$ to be Einstein.

\section{Main results}

Let $\left(B^{m}, \check{g}\right)$ and $\left(N^{n}, \hat{g}\right)$ be two Riemannian manifolds. And let $B \times{ }_{f} N:=(B \times N, \check{g}+f \hat{g})$ be the warped product of $B$ and $N$ by the positive smooth function $f$ on $B$. Let $\left\{\mathbf{b}_{i}\right\}_{i=1}^{m}$ and $\left\{\mathbf{n}_{\alpha}\right\}_{\alpha=1}^{n}$ be an (locally defined) orthonormal frames on $\left(B^{m}, \check{g}\right)$ and $\left(N^{n}, \hat{g}\right)$, respectively. We put $\mathbf{d}_{\alpha}:=f^{-1 / 2} \mathbf{n}_{\alpha}$. Then

$$
\left\{\left(\mathbf{b}_{i}, \mathbf{0}\right),\left(\mathbf{0}, \mathbf{d}_{\alpha}\right) \mid i=1,2, \cdots, m ; \alpha=1,2, \cdots, n\right\}
$$

is an (locally defined) orthonormal frame on $B \times{ }_{f} N$. From now on, we simply denote $\mathbf{b}_{i}:=\left(\mathbf{b}_{i}, \mathbf{0}\right), \mathbf{d}_{\alpha}:=\left(\mathbf{0}, \mathbf{d}_{\alpha}\right)$. Let $\left\{\check{\theta}^{i}\right\}_{i=1}^{m},\left\{\hat{\theta}^{\alpha}\right\}_{\alpha=1}^{n}$ 
and $\left\{\theta^{i}, \theta^{\alpha}\right\}_{i, \alpha}$ be the dual frames of $\left\{\mathbf{b}_{i}\right\}_{i=1}^{m},\left\{\mathbf{n}_{\alpha}\right\}_{\alpha=1}^{n}$ and $\left\{\mathbf{b}_{i}, \mathbf{d}_{\alpha}\right\}_{i, \alpha}$, respectively.

In general, the Riemannian connection $\nabla$ for the Riemannian metric $g$ on a Riemannian manifold $(M, g)$ is given by $([2,3,4])$

$$
\begin{aligned}
2 g\left(\nabla_{X} Y, Z\right)= & X g(Y, Z)+Y g(X, Z)-Z g(X, Y) \\
& -g(X,[Y, Z])-g(Y,[X, Z])+g(Z,[X, Y])
\end{aligned}
$$

for $X, Y, Z \in \mathfrak{X}(M)$.

Let $\check{\nabla}, \hat{\nabla}$ and $\nabla$ be the Levi-Civita connections on $(B, \check{g}),(N, \hat{g})$ and $B \times{ }_{f} N$, respectively. We introduce the notations $\check{\Gamma}_{j k}^{i}$ and $\hat{\Gamma}_{\beta \gamma}^{\alpha}$ such that $\check{\Gamma}_{j k}^{i}:=\check{\theta}^{i}\left(\check{\nabla}_{\mathbf{b}_{j}} \mathbf{b}_{k}\right)$ and $\hat{\Gamma}_{\beta \gamma}^{\alpha}:=\hat{\theta}^{\alpha}\left(\hat{\nabla}_{\mathbf{n}_{\beta}} \mathbf{n}_{\gamma}\right)$. We put

$$
\begin{aligned}
& \nabla_{\mathbf{b}_{i}} \mathbf{b}_{j}=\sum_{k=1}^{m} \Gamma_{i j}^{k} \mathbf{b}_{k}+\sum_{\alpha=1}^{n} \Gamma_{i j}^{\alpha} \mathbf{d}_{\alpha}, \quad \nabla_{\mathbf{b}_{i}} \mathbf{d}_{\alpha}=\sum_{k=1}^{m} \Gamma_{i \alpha}^{k} \mathbf{b}_{k}+\sum_{\gamma=1}^{n} \Gamma_{i \alpha}^{\gamma} \mathbf{d}_{\gamma}, \\
& \nabla_{\mathbf{d}_{\alpha} \mathbf{b}_{i}}=\sum_{k=1}^{m} \Gamma_{\alpha i}^{k} \mathbf{b}_{k}+\sum_{\gamma=1}^{n} \Gamma_{\alpha i}^{\gamma} \mathbf{d}_{\gamma}, \quad \nabla_{\mathbf{d}_{\alpha}} \mathbf{d}_{\beta}=\sum_{k=1}^{m} \Gamma_{\alpha \beta}^{k} \mathbf{b}_{k}+\sum_{\gamma=1}^{n} \Gamma_{\alpha \beta}^{\gamma} \mathbf{d}_{\gamma} .
\end{aligned}
$$

Using (2.2) and the above equations, we get

$$
\begin{aligned}
& \Gamma_{i j}^{k}=\check{\Gamma}_{i j}^{k}, \quad \Gamma_{i j}^{\alpha}=\Gamma_{i \alpha}^{j}=\Gamma_{i \alpha}^{\gamma}=\Gamma_{\alpha i}^{k}=0, \\
& \Gamma_{\alpha i}^{\beta}=-\Gamma_{\alpha \beta}^{i}=\frac{1}{2} f^{-1} \mathbf{b}_{i}(f) \delta_{\alpha \beta}, \quad \Gamma_{\alpha \beta}^{\gamma}=f^{-\frac{1}{2}} \hat{\Gamma}_{\alpha \beta}^{\gamma},
\end{aligned}
$$

that is

$$
\begin{aligned}
\nabla_{\mathbf{b}_{i}} \mathbf{b}_{j} & =\check{\nabla}_{\mathbf{b}_{i}} \mathbf{b}_{j}=\sum_{k=1}^{m} \check{\Gamma}_{i j}^{k} \mathbf{b}_{k}, \\
\nabla_{\mathbf{b}_{i}} \mathbf{d}_{\alpha} & =\mathbf{0}, \quad \nabla_{\mathbf{d}_{\alpha}} \mathbf{b}_{i}=-\frac{1}{2} f^{-1} \mathbf{b}_{i}(f) \mathbf{d}_{\alpha}, \\
\nabla_{\mathbf{d}_{\alpha}} \mathbf{d}_{\beta} & =-\frac{1}{2} \delta_{\alpha \beta} \sum_{i=1}^{m} \mathbf{b}_{i}(f) \mathbf{b}_{i}+f^{-\frac{1}{2}} \sum_{\gamma=1}^{n} \hat{\Gamma}_{\alpha \beta}^{\gamma} \mathbf{d}_{\gamma} \\
& =-\frac{1}{2} \delta_{\alpha \beta} \sum_{i=1}^{m} \mathbf{b}_{i}(f) \mathbf{b}_{i}+f^{-1} \hat{\nabla}_{\mathbf{n}_{\alpha}} \mathbf{n}_{\beta} .
\end{aligned}
$$


From (2.4) and $T^{\nabla}=\mathbf{0}$ (i.e. $\nabla$ is torsion-free), we have

$$
\left[\mathbf{b}_{i}, \mathbf{d}_{\alpha}\right]=-\frac{1}{2} f^{-1} \mathbf{b}_{i}(f) \mathbf{d}_{\alpha},\left[\mathbf{d}_{\alpha}, \mathbf{d}_{\beta}\right]=f^{-\frac{1}{2}} \sum_{\gamma=1}^{n}\left(\hat{\Gamma}_{\alpha \beta}^{\gamma}-\hat{\Gamma}_{\beta \alpha}^{\gamma}\right) \mathbf{d}_{\gamma}
$$

Let $(M, g),(N, h)$ be two Riemannian manifolds. Let $\phi: M \longrightarrow N$ be a smooth map. Let $E:=\phi^{-1} T N$ be the induced bundle by $\phi$ over $M$ of the tangent bundle $T N$ of $N$. We denote by $\Gamma(E)$, the space of all sections $V$ of $E$. We denote by $\nabla,{ }^{N} \nabla$ the Levi-Civita connections of $(M, g),(N, h)$, respectively. Then we give the induced connection $\tilde{\nabla}$ on $E$ by

$$
\left(\tilde{\nabla}_{X} V\right)_{x}:=\left.\frac{d}{d t}{ }^{N} P_{\phi(\gamma(t))}{ }^{-1} V_{\gamma(t)}\right|_{t=0}, \quad X \in \Gamma(T M), V \in \Gamma(E),
$$

where $x \in M, \gamma(t)$ is a curve through $x$ at $t=0$ whose tangent vector at $x$ is $X_{x}$, and ${ }^{N} P_{\phi(\gamma(t))}: T_{\phi(x)} N \longrightarrow T_{\phi(\gamma(t))} N$ is the parallel displacement along a curve $\phi(\gamma(s)), 0 \leq s \leq t$, given by the Levi-Civita connection ${ }^{N} \nabla$ of $(N, h)$.

For a $C^{\infty}$-map $\phi$ of an $m$-dimensional compact Riemannian manifold $(M, g)$ into another Riemannian manifold $(N, h)$, the following is well known (cf. $[5,7,9])$ : the map $\phi$ is harmonic if and only if $\tau(\phi)=0$ on $M$, where

$$
\tau(\phi):=\sum_{i=1}^{m}\left\{\tilde{\nabla}_{\mathbf{e}_{i}} \phi_{*} \mathbf{e}_{i}-\phi_{*}\left({ }^{M} \nabla_{\mathbf{e}_{i}} \mathbf{e}_{i}\right)\right\}
$$

for $\left\{\mathbf{e}_{i}\right\}_{i=1}^{m}$ an (locally defined) orthonormal frame on $(M, g)$.

From (2.6), we obtain the fact that a necessary and sufficient condition for the identity map $i:(B, \check{g}) \times(N, \hat{g}) \longrightarrow B \times{ }_{f} N$ to be harmonic is

$$
\sum_{\alpha=1}^{n}\left(\nabla_{\mathbf{n}_{\alpha}} \mathbf{n}_{\alpha}-\hat{\nabla}_{\mathbf{n}_{\alpha}} \mathbf{n}_{\alpha}\right)=\mathbf{0}
$$

On the other hand, we get

$$
\nabla_{\mathbf{n}_{\alpha}} \mathbf{n}_{\beta}=f \nabla_{\mathbf{d}_{\alpha}} \mathbf{d}_{\beta}=f \sum_{\gamma=1}^{n} \Gamma_{\alpha \beta}^{\gamma} \mathbf{d}_{\gamma} .
$$


Moreover, using (2.4), we have

$$
\hat{\nabla}_{\mathbf{n}_{\alpha}} \mathbf{n}_{\beta}=f\left\{\nabla_{\mathbf{d}_{\alpha}} \mathbf{d}_{\beta}+\frac{1}{2} \delta_{\alpha \beta} \sum_{i=1}^{m} \mathbf{b}_{i}(f) \mathbf{b}_{i}\right\} .
$$

By virtue of (2.3), (2.7), (2.8) and (2.9), we obtain

Proposition 2.1. Let $B \times_{f} N$ be the warped product Riemannian manifold of $\left(B^{m}, \check{g}\right)$ and $\left(N^{n}, \hat{g}\right)$, respectively. Assume that $i:(B, \check{g}) \times$ $(N, \hat{g}) \rightarrow B \times{ }_{f} N$ is the identity map as a map. Then, the following statements are equivalent:
(a) $i$ is harmonic;
(b) $\sum_{\alpha=1}^{n} \nabla_{\mathbf{n}_{\alpha}} \mathbf{n}_{\alpha}=\sum_{\alpha=1}^{n} \hat{\nabla}_{\mathbf{n}_{\alpha}} \mathbf{n}_{\alpha}$;
(c) $f$ is constant on $B$;
(d) $\sum_{\alpha=1}^{n} \Gamma_{\alpha \alpha}^{k}=0$ for each $k(k=1,2, \cdots, m)$.

From (2.3), (2.4), (2.5) and $R^{\nabla}(X, Y) Z:=\left[\nabla_{X}, \nabla_{Y}\right](Z)-\nabla_{[X, Y]} Z$ for $X, Y, Z \in \mathfrak{X}(B \times N)$, we get

$$
\begin{aligned}
R^{\nabla}\left(\mathbf{b}_{i}, \mathbf{b}_{j}\right) \mathbf{b}_{k}= & R^{\check{\nabla}}\left(\mathbf{b}_{i}, \mathbf{b}_{j}\right) \mathbf{b}_{k}, \quad R^{\nabla}\left(\mathbf{b}_{i}, \mathbf{b}_{j}\right) \mathbf{d}_{\alpha}=0, \\
R^{\nabla}\left(\mathbf{b}_{i}, \mathbf{d}_{\alpha}\right) \mathbf{b}_{j}= & \frac{1}{4}\left\{2 f^{-1} \mathbf{b}_{i}\left(\mathbf{b}_{j}(f)\right)-2 f^{-1} \sum_{k=1}^{m} \check{\Gamma}_{i j}^{k} \mathbf{b}_{k}(f)\right. \\
& \left.-f^{-2} \mathbf{b}_{i}(f) \mathbf{b}_{j}(f)\right\} \mathbf{d}_{\alpha}, \\
R^{\nabla}\left(\mathbf{b}_{i}, \mathbf{d}_{\alpha}\right) \mathbf{d}_{\beta}= & \frac{1}{4} \sum_{j=1}^{m}\left\{f^{-2} \mathbf{b}_{i}(f) \mathbf{b}_{j}(f)-2 f^{-1} \mathbf{b}_{i}\left(\mathbf{b}_{j}(f)\right)\right. \\
& \left.-2 f^{-1} \sum_{k=1}^{m} \check{\Gamma}_{i k}^{j} \mathbf{b}_{k}(f)\right\} \delta_{\alpha \beta} \mathbf{b}_{j}, \\
R^{\nabla}\left(\mathbf{d}_{\alpha}, \mathbf{d}_{\beta}\right) \mathbf{b}_{i}= & 0, \\
R^{\nabla}\left(\mathbf{d}_{\alpha}, \mathbf{d}_{\beta}\right) \mathbf{d}_{\gamma}= & \hat{R}\left(\mathbf{d}_{\alpha}, \mathbf{d}_{\beta}\right) \mathbf{d}_{\gamma} \\
& +\frac{1}{4} f^{-2} \sum_{i=1}^{m}\left(\mathbf{b}_{i}(f)\right)^{2}\left(\delta_{\alpha \gamma} \mathbf{d}_{\beta}-\delta_{\beta \gamma} \mathbf{d}_{\alpha}\right) .
\end{aligned}
$$

The Ricci tensor field $\operatorname{Ric}^{\nabla}$ of type $(0,2)$ is defined by

(2.11) $\operatorname{Ric}^{\nabla}(Y, Z):=\operatorname{trace}\left\{X \rightarrow R^{\nabla}(X, Y) Z\right\} \quad(X, Y, Z \in \mathfrak{X}(B \times N))$. 
Now, we assume $(B, \check{g})$ and $(N, \hat{g})$ are Einstein manifolds such that

$$
\operatorname{Ric} c^{\check{\nabla}}=\check{c} \check{g}, \quad \operatorname{Ric} \hat{\nabla}=\hat{c} \hat{g} .
$$

From (2.10), (2.11) and (2.12), we have

$$
\begin{aligned}
\operatorname{Ric}^{\nabla}\left(\mathbf{b}_{i}, \mathbf{b}_{j}\right)= & \check{c} \delta_{i j}+\frac{n}{4}\left\{f^{-2} \mathbf{b}_{i}(f) \mathbf{b}_{j}(f)\right. \\
& \left.-2 f^{-1} \mathbf{b}_{i}\left(\mathbf{b}_{j}(f)\right)+2 f^{-1} \sum_{k=1}^{m} \check{\Gamma}_{i j}^{k} \mathbf{b}_{k}(f)\right\}, \\
\operatorname{Ric}^{\nabla}\left(\mathbf{b}_{i}, \mathbf{d}_{\alpha}\right) & =0, \\
\operatorname{Ric}^{\nabla}\left(\mathbf{d}_{\alpha}, \mathbf{d}_{\beta}\right) & =\frac{1}{4}\left\{(2-n) f^{-2} \sum_{i=1}^{m}\left(\mathbf{b}_{i}(f)\right)^{2}\right. \\
& \left.-2 f^{-1} \sum_{i=1}^{m} \mathbf{b}_{i}\left(\mathbf{b}_{i}(f)\right)+2 f^{-1} \sum_{i, k=1}^{n} \check{\Gamma}_{i i}^{k} \mathbf{b}_{k}(f)\right\} \delta_{\alpha \beta} \\
& +f^{-1} \hat{c} \delta_{\alpha \beta} .
\end{aligned}
$$

Then, we obtain the following

Theorem 2.2. Let $\left(B^{m}, \check{g}\right)$ and $\left(N^{n}, \hat{g}\right)$ be Einstein manifolds such that $\operatorname{Ric}^{\check{\nabla}}=\check{c} \check{g}$ and $R i c^{\hat{\nabla}}=\hat{c} \hat{g}$. Suppose that the identity map $i:(B \times N, \check{g}+\hat{g}) \rightarrow B \times{ }_{f} N$ is harmonic. Then $B \times{ }_{f} N$ be Einstein if and only if $\hat{c}=f \check{c}$.

Proof. Assume $i$ is harmonic. Then, from Proposition 2.1 we obtain the function $f$ is constant on $B$.

Suppose that $B \times{ }_{f} N$ is Einstein such that $R i c^{\nabla}=c g$ for a constant c. Then we get from the first formula of (2.13)

$$
c \delta_{j k}=\operatorname{Ric}^{\nabla}\left(\mathbf{b}_{j}, \mathbf{b}_{k}\right)=\check{c} \delta_{j k} .
$$

Moreover, we have from the last formula of (2.13)

$$
c \delta_{\alpha \beta}=\operatorname{Ric}{ }^{\nabla}\left(\mathbf{d}_{\alpha}, \mathbf{d}_{\beta}\right)=f^{-1} \hat{c} \delta_{\alpha \beta} .
$$

By virtue of (2.14) and (2.15), we obtain

$$
c=\check{c}=f^{-1} \hat{c} .
$$


Conversely, let $\hat{c}=f \check{c}$. Then we get from (2.13)

$$
\left\{\begin{array}{l}
\operatorname{Ric}^{\nabla}\left(\mathbf{b}_{i}, \mathbf{b}_{j}\right)=\check{c} \delta_{i j}=\check{c} g\left(\mathbf{b}_{i}, \mathbf{b}_{j}\right) \\
\operatorname{Ric}^{\nabla}\left(\mathbf{b}_{i}, \mathbf{d}_{\alpha}\right)=0=c g\left(\mathbf{b}_{i}, \mathbf{d}_{\alpha}\right) \\
\operatorname{Ric}^{\nabla}\left(\mathbf{d}_{\alpha}, \mathbf{d}_{\beta}\right)=f^{-1} \hat{c} \delta_{\alpha \beta}=\check{c} g\left(\mathbf{d}_{\alpha}, \mathbf{d}_{\beta}\right)
\end{array}\right.
$$

since $f$ is constant. Hence $R i c^{\nabla}=\check{c} g$, and then $B \times_{f} N$ is Einstein.

The Laplacian $\Delta_{g}$ of an $n$-dimensional Riemannian manifold $(M, g)$ is given by $\Delta_{g}:=-\sum_{i=1}^{n}\left(e_{i}{ }^{2}-\nabla_{e_{i}} e_{i}\right)$, where $\left\{e_{i}\right\}_{i=1}^{n}$ is an (locally defined) orthonormal frame on $(M, g))$. We denote the spectrum $\operatorname{Spec}\left(\Delta_{g}\right)$ of $\Delta_{g}$ of a compact Riemannian manifold $(M, g)$ is denoted by $([8,9])$

$$
\operatorname{Spec}\left(\Delta_{g}\right)=\left\{\lambda_{0}(g)=0 \leq \lambda_{1}(g) \leq \lambda_{2}(g) \leq \cdots \leq \uparrow \infty\right\} .
$$

On the other hand, we get the following

Theorem 2.3. Let $\left(B^{m}, \check{g}\right)$ and $\left(N^{n}, \hat{g}\right)$ be Einstein manifolds such that $R i c^{\check{\nabla}}=\check{c} \check{g}$ and $R i c^{\hat{\nabla}}=\hat{c} \hat{g}$. Then $B \times_{f} N$ is Einstein if and only if

$$
\begin{gathered}
4 \check{c}+n\left\{f^{-2}\left(\mathbf{b}_{j}(f)\right)^{2}-2 f^{-1} \mathbf{b}_{j}\left(\mathbf{b}_{j}(f)\right)+2 f^{-1} \sum_{i=1}^{m} \check{\Gamma}_{j j}^{i} \mathbf{b}_{i}(f)\right\} \\
=2 f^{-1}\left(2 \hat{c}+\Delta_{\check{g}} f\right)+(2-n) f^{-2}\|d f\|_{\check{g}}^{2}
\end{gathered}
$$

for each $j$, and

$$
\mathbf{b}_{j}(f) \mathbf{b}_{k}(f)-2 f \mathbf{b}_{j}\left(\mathbf{b}_{k}(f)\right)+2 f \sum_{i=1}^{m} \check{\Gamma}_{j k}^{i} \mathbf{b}_{i}(f)=0
$$

for $j, k(j \neq k)$ are hold.

Proof. The warped product manifold $B \times_{f} N$ is Einstein if and only if

$$
R i c^{\nabla}=c g
$$


for some constant $c$. From (2.13) and (2.17), we get the fact that (2.17) holds if and only if

$$
\begin{gathered}
4 c \delta_{j k}=4 \check{c} \delta_{j k}+n\left\{f^{-2} \mathbf{b}_{j}(f) \mathbf{b}_{k}(f)-2 f^{-1} \mathbf{b}_{j}\left(\mathbf{b}_{k}(f)\right)\right. \\
\left.+2 f^{-1} \sum_{i=1}^{m} \check{\Gamma}_{j k}^{i} \mathbf{b}_{i}(f)\right\} \\
4 c=2 f^{-1}\left(2 \hat{c}+\Delta_{\check{g}} f\right)+(2-n) f^{-2}\|d f\|_{\check{g}}^{2} .
\end{gathered}
$$

In order to show the stability of Einstein manifolds, we introduce R.T. Smith's stability theorem :

Theorem 2.4[9]. Let $(M, g)$ be a compact Einstein Riemannian manifold such that the Ricci tensor $\rho$ satisfies $\rho=c g$. Then, the identity map of $(M, g)$ is weakly stable if and only if the first positive eigenvalue of the Laplacian $\Delta_{g}$ acting on $C^{\infty}(M), \lambda_{1}(g)$, satisfies $\lambda_{1}(g) \geq 2 c$.

If, for a Riemannian manifold $(M, g)$, the identity map of $(M, g)$ is stable (resp. unstable) as a harmonic map, then the manifold $(M, g)$ is said to be stable (resp. unstable).

Now, we obtain the following

Theorem 2.5. Let $\left(B^{m}, \check{g}\right)$ and $\left(N^{n}, \hat{g}\right)$ be Einstein manifolds such that $m \neq n, R i c^{\check{\nabla}}=\check{c} \check{g}$ and $\operatorname{Ric} \hat{\nabla}=\hat{c} \hat{g}$. Suppose that $B \times{ }_{f} N$ is Einstein. Then, the warped product manifold $B \times_{f} N$ is weakly stable if and only if

$$
\lambda_{1}(g) \geq \frac{2}{m-n}\left\{m \check{c}-n f^{-1} \hat{c}+\frac{1}{4} f^{-2}\left(n^{2}-n\right)\|d f\|_{\check{g}}^{2}\right\},
$$

where $\lambda_{1}(g)$ is the first positive eigenvalue of the Laplacian $\Delta_{g}$ of the warped product manifold $B \times{ }_{f} N$.

Proof. Assume $(B, \check{g}),(N, \hat{g})$ and $B \times_{f} N$ are Einstein. Then, summing over $j$ which is appeared in the condition (i) of Theorem 2.3, we 
have

$$
\begin{aligned}
4 m \check{c}+n \sum_{j=1}^{m}\left\{f^{-2}\left(\mathbf{b}_{j}(f)\right)^{2}-2 f^{-1} \mathbf{b}_{j}\left(\mathbf{b}_{j}(f)\right)+2 f^{-1} \sum_{k=1}^{m} \check{\Gamma}_{j j}^{k} \mathbf{b}_{k}(f)\right\} \\
=m\left\{2 f^{-1}\left(2 \hat{c}+\Delta_{\check{g}} f\right)-(n-2) f^{-2}\|d f\|_{\check{g}}^{2}\right\} .
\end{aligned}
$$

From the above equation, we obtain

$$
\begin{aligned}
4 m \check{c}+ & n\left\{f^{-2}|| d f \|_{\check{g}}^{2}+2 f^{-1} \Delta_{\check{g}} f\right\} \\
& =m\left\{2 f^{-1}\left(2 \hat{c}+\Delta_{\check{g}} f\right)-(n-2) f^{-2}\|d f\|_{\check{g}}^{2}\right\},
\end{aligned}
$$

and hence

(2.19) $2(m-n) f^{-1} \Delta_{\check{g}} f=4 m\left(\check{c}-f^{-1} \hat{c}\right)+\{m(n-2)+n\} f^{-2}\|d f\|_{\check{g}}^{2}$.

From the fact that $B \times{ }_{f} N$ is Einstein, we get

$$
R i c^{\nabla}=c g \text { for some constant } c .
$$

By the help of (2.19),(2.20) and the second formula of (2.18), we obtain

$$
c=(m-n)^{-1}\left\{m \check{c}-n f^{-1} \hat{c}+\frac{1}{4} f^{-2}\left(n^{2}-n\right)\|d f\|_{\check{g}}^{2}\right\} .
$$

By virtue of (2.21) and Theorem 2.4, the proof of this theorem is completed.

By the help of Theorems 2.4 and 2.5, we get

Corollary 2.6. Let $\left(B^{m}, \check{g}\right)$ and $\left(N^{n}, \hat{g}\right)$ be Einstein manifolds such that $m \neq n, R i c^{\check{\nabla}}=\check{c} \check{g}$ and $R i c^{\hat{\nabla}}=\hat{c} \hat{g}$. Suppose that $B \times{ }_{f} N$ is Einstein. Then, if

$$
\frac{1}{m-n}\left\{m \check{c}-n f^{-1} \hat{c}+\frac{1}{4} f^{-2}\left(n^{2}-n\right)\|d f\|_{\check{g}}^{2}\right\} \leq 0,
$$

the warped product manifold $B \times{ }_{f} N$ is stable. 


\section{References}

[1] A. L. Besse, Einstein Manifolds, Springer-Verlag, Berlin (1987).

[2] S. Helgason, Differential Geometry, Lie Groups and Symmetric Spaces, Academic Press, New York (1978).

[3] S. Kobayashi and K. Nomizu, Foundations of Differential Geometry, Vol. 1, Wiley-Interscience, New York (1963).

[4] K. Nomizu and T. Sasaki, Affine Differential Geometry - Geometry of Affine Immersions, Cambridge Univ. Press (1994).

[5] J.-S. Park. Harmonic inner automorphisms of compact connected semisimple Lie groups, Tohoku Math. J. 42 (1990), 83-91.

[6] J.-S. Park. Critical homogeneous metrics on the Heisenberg manifold, Inter. Inform. Sci. 11 (2005), 31-34.

[7] J.-S. Park and W. T. Oh, The Abbena-Thurston manifold as a critical point, Can. Math. Bull. 39 (1996), 352-359.

[8] N. Shimakura, Elliptic Partial Differential Operators of Elliptic Type, Transl. Math. Monographs, Vol. 99, Amer. Math. Soc., Providence, RI, 1991.

[9] H. Urakawa, Calculus of Variations and Harmonic Maps, Transl. Math. Monographs, Vol. 99, Amer. Math. Soc., Providence, RI, 1993.

Yong-Soo Pyo

Department of Applied Mathematics,

Pukyong National University,

Pusan 608-737, Korea

E-mail: yspyo@pknu.ac.kr

Hyun Woong Kim

Department of Applied Mathematics,

Pukyong National University,

Busan 608-737, Korea

E-mail: 0127woong@hanmail.net

Joon-Sik Park

Department of Mathematics,

Pusan University of Foreign Studies,

Busan 608-738, Korea

E-mail: iohpark@pufs.ac.kr 